\title{
Quantitative Evaluation of Artefact Reduction by an Optimized Specimen Orientation for Metrology based on Industrial Computed Tomography
}

\author{
Manuel Kaufmann ${ }^{1}$, Ira Effenberger ${ }^{1}$ \\ 1 Fraunhofer Institute for Manufacturing Engineering and Automation IPA \\ Nobelstr. 12, 70569 Stuttgart, Germany \\ manuel.kaufmann@ipa.fraunhofer.de
}

\begin{abstract}
Summary:
Industrial Computed Tomography is used to solve metrological tasks in quality assurance. Although CT has many advantages like complete and non-destructive data acquisition, artefacts can occur depending on the part's material and geometry. Moreover the extent of scanning artefacts strongly depends on the specimen's orientation. By a raycasting approach, the homogeneity of penetration lengths is determined and maximized in order to find the optimal orientation. The reduction of artefacts is assessed by point-based quality metrics. The results show a local contrast improvement of $80 \%$.
\end{abstract}

Keywords: Industrial Computed Tomography, Artefacts, Reduction, Quality

\section{Introduction and Problem Setting}

Industrial Computed Tomography (CT) is well established in industry both for inspection and metrology tasks. The concept of the CT is based on capturing a set of x-ray projection images, usually over a full rotation of the object, that are reconstructed to a $3 \mathrm{D}$ volume. However due to interaction between $x$-ray photons and the observed specimen, in the reconstruction algorithm an assumed linear x-ray propagation is violated. As a consequence artefacts occur that are defined as discrepancies between the real attenuation coefficients of the specimen and the reconstructed values [1]. Figure 1 shows typical artefacts for dense materials such as Aluminum for the analyzed use case part. Here streaks between edges and high-contrast regions, contrast loss and cupping artefacts occur.
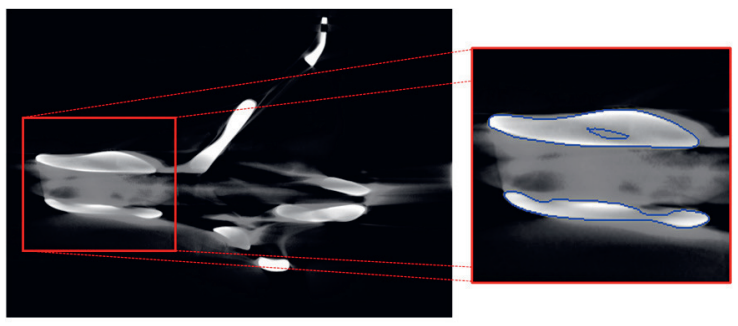

Fig. 1. Artefacts and surface extraction depicted in the detail view, which is denoted with blue lines.

Thus, the possibility to detect small geometrical features such as inner defects and the accuracy of a surface determination is impaired by these artefacts. In range of photon energies typically occurring for CT, beam hardening and Compton scattering are the main effects of photon-matter interaction. According to [2], both effects are influenced by the specimen's material, the CT parameters defining the $x$-ray spectrum, the specimen's geometry and its position and orientation within the x-ray beam.

\section{Aim and Used Approach}

In our work, the influence of the specimen orientation on the extent of artefacts is researched. Therefore, two orientations of a use case part, a best and a worst orientation, are studied. In order to determine an optimal specimen orientation, a raycasting approach is incorporated to gather the local penetration lengths. In the state of the art [3], a minimization of penetration lengths is performed. However in our approach, the optimal orientation is gathered by maximizing the homogeneity of penetration lengths. Figure 2 gives an example for less and more homogeneous penetration lengths, where in the prior case a higher grey value dynamic occurs.

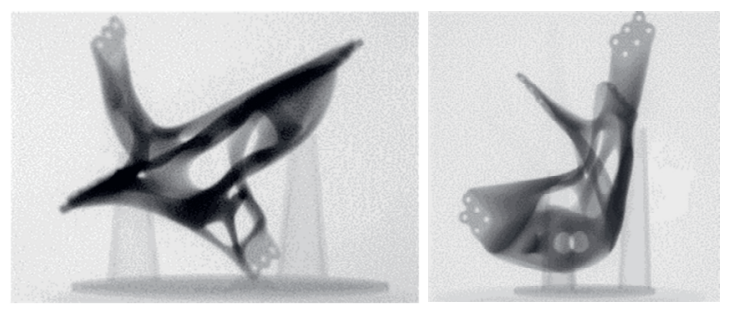

Fig. 2. Projection images of less (left image) and more homogeneous penetration lengths (right image). 
The geometrical setup is shown in Figure 3 , where the optimization variables $\phi$ and $\theta$ are depicted.

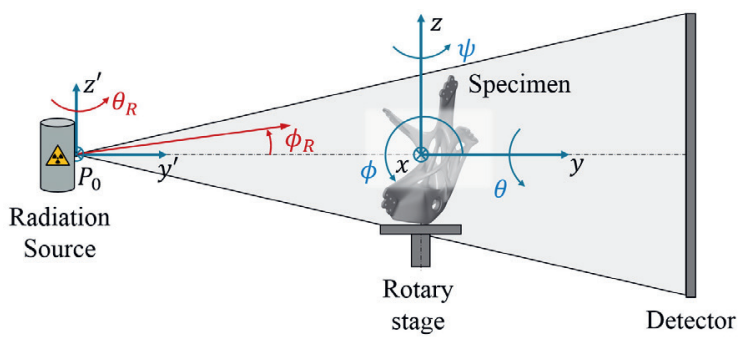

Fig. 3. Set-up for specimen orientation optimization.

\section{Quantification of the Artefact Reduction by Point-based Quality Metrics}

In order to evaluate the extent of artefact reduction, quality metrics for both best and worst case orientations are compared. As quality metrics, the Michaelson contrast $\mathrm{C}_{\mathrm{M}}$ and parameters of a sigmoid function approximation are considered. The contrast $\mathrm{C}_{M}$ is computed according to

$\mathrm{C}_{\mathrm{M}}=\frac{\mathrm{I}_{\max }-\mathrm{I}_{\min }}{\mathrm{I}_{\max }+\mathrm{I}_{\min }}$ with $0 \leq \mathrm{C}_{\mathrm{M}} \leq 1$.

Here, $I_{\max }$ and $I_{\min }$ are the maximum and minimum grey values in the reconstructed volume, respectively. The contrast $C_{M}$ is gathered for each surface point $P$ of the determined ISO 50 surface. A higher scan quality is associated with a higher contrast value. Furthermore, the parameters $S_{0}$ and $S_{1}$ of a sigmoid function are approximated for each grey value profile through the surface point $P$ and along the corresponding surface normal $\vec{n}$. The sigmoid function is defined according to

$\operatorname{sig}(q)=S_{1} \cdot \frac{1}{1+e^{-q}}+S_{0}$

where $q$ denotes a control variable along the surface normal $\vec{n}$. A smaller $S_{0}$ value (grey value offset) indicates smaller grey values of the air surrounding the specimen and thus implies the absence of surrounding artefacts. An increased $S_{1}$ value (grey value slope) indicates a steeper grey value transition between air and material and implies a better separation of material from background.

\section{Results}

The considered quality metrics are studied for a 3D printed aircraft bracket part made from an Aluminum alloy. Both the best and worst case orientations are determined by deploying the described raycasting approach. The worst case orientation in contrast is determined by minimizing the projection image homogeneity.

In Figures 4 and 5 , the local contrast $\mathrm{C}_{\mathrm{M}}$ is shown for both orientations. For most regions on the scanned specimen, the contrast is strongly improved by scanning in the optimized orientation. On average, the contrast is increased by $79 \%$. The grey value offset $S_{0}$ is decreased by $31 \%$ and the slope $S_{1}$ is increased by $36 \%$. These numbers allow the conclusion that a strong artefact reduction due to an optimized orientation was achieved. By evaluating point-based quality metrics, a significant improvement of the scan quality is shown for an optimized orientation of a part with complex geometry. Artefacts may be shifted to other regions on the specimen indeed, whereby quality values of former high-quality regions become smaller. For most regions however, the reduction of artefacts eventually improves the results of voxel-based evaluations like surface determinations and defect analyses.

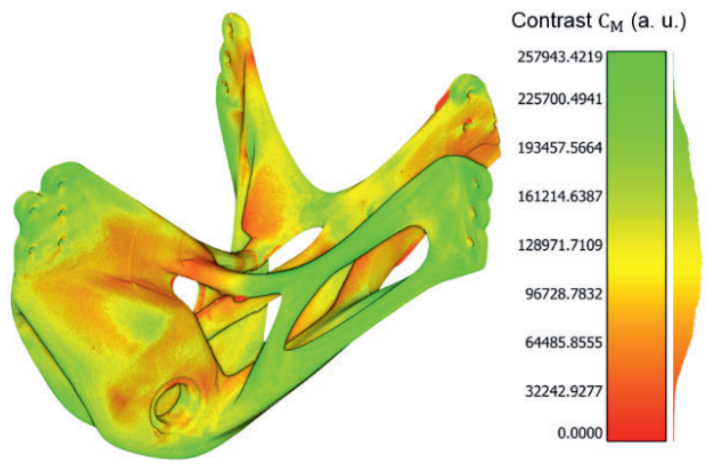

Fig. 4. Contrast values for the analyzed specimen at worst orientation.

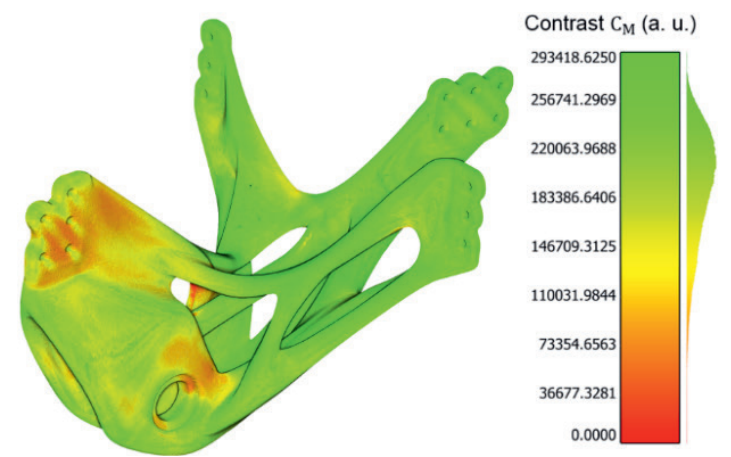

Fig. 5. Contrast values for the analyzed specimen at best orientation.

\section{References}

[1] J. Hsieh, Computed tomography. Principles, design, artifacts, and recent advances. 2nd ed., Hoboken: Wiley Interscience (2009); SPIE Press.

[2] A. Amirkhanov et al., Visual optimality and stability analysis of 3DCT scan positions. In: Trans. Vis. Comput. Graph. 16 (6), S. 1477-1486 (2010); doi: 10.1109/TVCG.2010.214.

[3] C. Heinzl et al., Fast Estimation of Optimal Specimen Placements in 3D X-ray Computed Tomography. In: International Symposium on Digital Industrial Radiology and Computed Tomography (2010). 